\title{
De-formazioni in corso. Mutamenti nella struttura religiosa del cattolicesimo italiano
}

\author{
Luca Diotallevi
}

During the last forty years Italian Catholicism has experienced a deep change. It can be directly observed focusing on the Catholic religious authority structure in Italy (CRASI). It shows both a quantitative as well as a morphological change. From the quantitative point of view CRASI has lost a lot of organizations and a lot of clerical (male and female) human resources. From the morphological point of view the CRASI has become more centralized, more verticalized and - at the same time - its top/down channels are now multiplied and strongly diversified each from the other. Finally, the change in the CRASI has been interwined with deep changes occurring in nonreligious areas of Italian Catholicism. Among others, Italian political Catholicism offers only the best example of the radical demise in the extra religious relevance of Italian Catholicism.

Luca Diotallevi is Professor of Sociology at Roma Tre University.

Secondo Max Weber "per chiesa si deve intendere una impresa istituzionale di carattere ierocratico identificata dalla capacità di concedere o di rifiutare beni sacri (NB.: esercitando la cosiddetta "coercizione ierocratica”) nella quale - e nella misura in cui - l'apparato amministrativo avanza la pretesa di monopolio della coercizione ierocratica legittima" (Weber 1995, p. 53). In modo particolarmente efficace, e adeguato a contesti esposti alle tensioni della secolarizzazione, M. Chaves (1994) ha impiegato le più recenti risorse della sociologia della organizzazione per analizzare le forme e i problemi di quel particolare tipo di apparato amministrativo (1993). Esso è stato isolato e studiato come "struttura dell'autorità religiosa" (religious authority structure). Con tale concetto si intende dunque semplicemente una componente del sistema religioso e di una tradizione religiosa.

Nel contributo che segue ${ }^{1}$ si cercherà di mettere in luce le $d e$-formazioni che la struttura dell'autorità religiosa cattolica in Italia ha sperimentato e sta sperimentando. Queste saranno fatte emergere confrontando idealmente due fotografie della medesima struttura: una scattata ai giorni nostri e una scattata all'inizio degli anni '70. Per valutare i risultati di questa operazione è importante tener presente che essa non intende considerare l'intera multiforme presenza e influ-

1 Per un quadro analitico più esteso e dettagliato si veda Diotallevi 2014c; per una interpretazione d'insieme: Diotallevi 2014b; per una interpretazione delle trasformazioni che più direttamente interessano il clero: Diotallevi 2014. 
enza del cattolicesimo nella vita della società italiana, dalla quale non avrebbe senso, ad esempio, escludere fenomeni come il cattolicesimo politico o le correnti artistiche che del cattolicesimo sono state espressione nei rispettivi ambiti, diversi da quello religioso. Inoltre, non si intende neppure considerare in tutte le sue diverse manifestazioni il solo cattolicesimo religioso. Di questo, infatti, ci si limiterà a considerare, e ovviamente solo da un punto di vista sociologico, la struttura organizzata dell'autorità (religiosa, appunto) che vi si esercita. Ancor più precisamente - per dirla con Luhmann (1985) - qui se ne analizzeranno principalmente due componenti: la forma di alcune delle più rilevanti organizzazioni di quella struttura e poi alcune delle principali caratteristiche del personale come risorsa a disposizione di quelle organizzazioni.

In estrema sintesi, il presente contributo cerca di fornire argomenti a sostegno della seguente ipotesi. Dal 1970 a oggi, la struttura religiosa del cattolicesimo italiano ha conosciuto profonde trasformazioni (a cominciare da un forte processo di centralizzazione e verticalizzazione). L'effetto di queste trasformazioni è stato duplice. Per un verso esse hanno contribuito, certo non da sole, a rallentare rispetto ad altri casi nazionali comparabili - la crisi del cattolicesimo religioso; per altro verso hanno compromesso alcuni tratti essenziali della forma-chiesa tipica del cattolicesimo. Per quest'ultima ragione, e senza alcun accento valutativo, è possibile comprendere quelle stesse trasformazioni in termini di de-formazioni. Così, al termine del periodo considerato, il cattolicesimo italiano si presenta più piccolo e più religioso, meno influente in ambiti extra-religiosi e più esposto al fascino del nuovo religious booming. Si trova insomma di fronte a una forte contraddizione: da una parte le esigenze della forma religiosa vincente (la "low intensity religion") e dall'altra le istanze interne del cristianesimo e della sua tradizione cattolica.

\section{Le strutture}

Ricorrendo a metafore spaziali, si può dire che tra l'inizio e la fine del periodo considerato $(i)$ la base di questa struttura ha conosciuto un restringimento o, meglio ancora, una riduzione della capillarità della propria presenza sul territorio. Al contempo, (ii) quello che all'inizio era un poco sviluppato elemento di raccordo tra le diocesi (la Conferenza Episcopale Italiana), al termine ha visto enormemente accresciuto il proprio potere. Quest'ultima trasformazione è proceduta a tal punto che ormai la C.E.I. è percepita come una sorta di centro e di vertice, anche se non assoluti, dell'organizzazione religiosa cattolica nel paese. In breve: al termine dei quarant'anni considerati, la struttura dell'autorità religiosa cattolica in Italia manifesta - tra gli altri - il duplice segno $(i)$ di una forte verticalizzazione e di una ancor più forte centralizzazione, per un verso, e, per altro verso, (ii) quello di una contrazione (forse non solo) quantitativa dei propri terminali territoriali. 
Nel corso del quarantennio preso in considerazione, l'élite ecclesiastica italiana perde il controllo del "trono di Pietro". Da ormai quasi trentacinque anni (precisamente dall'ottobre del 1978), infatti, e per la prima volta dopo più di quattro secoli, non è più un italiano ad essere Papa. Come era prevedibile, questo cambiamento non ha significato assolutamente una riduzione dell'intensità del rapporto tra il vescovo di Roma e le altre chiese particolari presenti in Italia, ma un profondo cambiamento delle forme e dei contenuti di questo rapporto. Difatti era probabilmente dai tempi di Pio XI (pontefice dal 1922 al 1939) che un papa non elaborava ex novo e non perseguiva con tanta sistematicità un progetto di governo religioso sull'Italia come si è verificato con Karol Wojtyła: Giovanni Paolo II dal 1978 al 2005 (Acerbi 2006). Come conseguenza di ciò, durante il papato wojtyliano si verificheranno molte innovazioni relative alla struttura della autorità religiosa del cattolicesimo italiano, e degli effetti di almeno alcune di queste sarà necessario trattare. Sin dall'inizio, però, è opportuno chiarire che del complesso movimento di attenuazione dell'influenza delle élites e delle istituzioni ecclesiastiche italiane sul vertice della curia vaticana - per quanto contraddittorio possa a prima vista apparire - è parte integrante l'accrescimento del potere a disposizione delle organizzazioni centrali della Conferenza Episcopale Italiana. Queste ultime divengono prima strumenti e poi partner nella elaborazione e nella esecuzione del nuovo programma di governo da Roma del cattolicesimo religioso italiano. In proposito è sufficiente ricordare le originali modalità di elezione dei vertici della CEI (per lo meno quelle formalmente in vigore sino agli inizi del 2014). Solo nel caso di questa conferenza episcopale nazionale (e in pochissimi altri di minore rilievo) i vertici non sono eletti dai vescovi delle diocesi di quel paese, bensì nominati dal Pontefice. Per di più, e per molti decenni, il ruolo di Presidente della C.E.I. ha personalmente coinciso con quello di vicario del Papa per la diocesi di Roma.

Dunque, per intendere correttamente il significato del processo di centralizzazione di cui si tratterà tra breve, occorre non cedere all'ingenuità di pensare che i vertici di questa organizzazione abbiano sostituito (in un qualsiasi senso) il ruolo del Papa rispetto al cattolicesimo religioso italiano. ${ }^{2} \mathrm{~L}$ 'impatto del processo di centralizzazione-verticalizzazione sul livello regionale trasforma sempre più spesso il ruolo delle conferenze episcopali regionali da supporto alla vita dio-

2 Anche se in questo Paese la intensità del processo di centralizzazione tocca livelli raramente o mai raggiunti altrove, esso ha caratteri di generalità tali da far sorgere un inedito dibattito tra due teologi esponenti di primo rango del "governo" wojtyłiano: gli allora autorevoli cardinali di curia Kasper e Ratzinger. Per altro, è proprio di questa fine 2013 la notizia che il nuovo Papa, l'argentino Jorge M. Bergoglio, ha chiesto alla C.E.I. una sollecita modifica statutaria che cambi le forme di elezione dei vertici della stessa indicando come obiettivo una qualche forma di elezione diretta di questi da parte della assemblea dei vescovi delle diocesi italiane ed una maggiore valorizzazione delle conferenze episcopali regionali. 
cesana a "cinghia di trasmissione" tra "centro" (i vertici della C.E.I.) e "periferia" (le organizzazioni diocesane) della struttura della autorità religiosa.

\subsection{Le diocesi}

Nella prima metà degli anni '80 le diocesi italiane vengono ridotte quasi di un quarto. Esse passano da 285 circa a poco più di 220 [A].

Per contro, a partire dalla seconda metà dello stesso decennio, l'organizzazione centrale della CEI. (operativa da decenni, ma che aveva sempre avuto dimensioni e competenze limitatissime e che aveva potuto contare su risorse materiali e simboliche molto modeste) conosce uno sviluppo più che esponenziale. Descriverne i dettagli eccede del tutto il compito di questo contributo. Qui è più che sufficiente indicare l'ordine di grandezza di questo incremento di potere. Il dato finanziario [I] si presta bene a questo scopo.

Tra il 1990 e il 2010 le organizzazioni ecclesiastiche italiane hanno potuto contare su un flusso crescente di risorse provenienti dalle scelte dei contribuenti in ordine alla destinazione dell'“otto per mille". Per effetto di queste decisioni tali risorse a disposizione delle organizzazioni ecclesiastiche italiane sono passate dai 209,7 milioni di Euro del $1990^{3}$ ai 1.032,7 milioni del 2013 [I].

Anche solo sulla base di questo dato elementare non è difficile immaginare quale incremento di potere abbiano conosciuto gli organismi centrali della C.E.I.. A questi organismi, infatti, era stato attribuito in sede concordataria il compito di istruire e di amministrare tutto quanto concerne la raccolta e l'impiego di tale tipo di risorse, salvo la annuale approvazione del bilancio da parte della assemblea plenaria dei vescovi delle diocesi italiane. ${ }^{4}$

Di per sé non era il crearsi di queste nuove e così abbondanti risorse a mettere in discussione il grado di autonomia economica (e non solo economica) raggiunto sino al 1984 dalle diocesi rispetto al vertice della CEI. La spiegazione del processo di verticalizzazione-accentramento va ricercata, tra l'altro, nelle forme assunte e negli scopi perseguiti dall'amministrazione di queste nuove e ingentissime risorse. Le forme concrete attraverso le quali si è determinato il processo di centralizzazione-verticalizzazione di cui stiamo parlando non hanno inficiato i principi giuridici alla base del concordato del 1984. Né di per sé hanno violato le regole di trasparenza e di pubblicità nell'amministrazione economica dei beni della Chiesa che invece raggiunge in questi casi standard elevatissimi e con rari altri esempi (anche se si allarga lo sguardo allo scenario internazionale). Semplicemente, se le

3 Nei primi anni si trattava di anticipi, in attesa che il sistema entrasse a regime. Visto il volume inatteso delle firme a favore della Chiesa cattolica, quegli anticipi si rivelarono ampiamente sottostimati.

4 Tra l'altro, una delle caratteristiche dello Statuto della CEI al momento vigente è il vincolo diretto presidente-amministratore (cfr. art.35 e art.98 del Regolamento). 
scelte procedurali e materiali di gestione di tali risorse non contraddicono né quei principi né quegli standard, esse non sono state certo meccanicamente dedotte da questi. Altre sarebbero state, e sono, altrettanto compatibili con gli stessi principi e gli stessi standard. ${ }^{5}$

Ciò che di fatto si verifica, e che giustifica ampiamente l'impiego di categorie come quella di "centralizzazione", è $(a)$ il progressivo aumento della dipendenza della "periferia" dal "centro", delle diocesi dalla CEI, e $(b)$ la quota notevole e crescente delle risorse che il "centro" trattiene per sé. È per questa via che i vertici della CEI hanno potuto affiancare a una forte investitura ricevuta dal pontefice e agli effetti di alcuni successi (cfr. ad es. Diotallevi 2007), una inedita, notevole e continuamente crescente disponibilità di risorse finanziarie.

L'andamento della ripartizione dei fondi provenienti dalle scelte "otto per mille" fornisce l'indicazione sintetica di cui abbiamo bisogno in questa sede (cfr. " $a$ "). L'insieme dei fondi assegnati in sede CEI alle diocesi e dei fondi destinati al sostentamento del clero (che nella quasi totalità presta servizio nelle e per le

5 Credo sia sufficiente fare due esempi. Non solo la quota a posteriori destinata alle diocesi, ma tutti i fondi "otto per mille" potevano essere ripartiti tra le diocesi, lasciando poi a queste di decidere come e quanto contribuire alla vita e alle funzioni degli organismi centrali della CEI. Per questa via è difficile essere certi che sarebbero stati conseguiti gli stessi notevoli risultati, ma, chiaramente, i rapporti di forza tra centro e periferia sarebbero risultati ben diversi da quelli attuali. Non si sarebbe verificato, cioè, che un organismo (i vertici CEI) in linea di principio di valore enormemente inferiore rispetto a ciascuna delle singole chiese particolari (o "diocesi") avesse finito per avere invece su queste tanto potere di influenza, di indirizzo e di controllo. Secondo esempio. Le altre quattro fonti del sostentamento del clero, oltre il gettito proveniente dall'"otto per mille", sono basate - a differenza di quest'ultima - non sulla espressione puntuale nel tempo della "identificazione religiosa", ma su complessi e duraturi meccanismi di partecipazione, come è noto socialmente molto più costosi. Nello specifico si tratta delle "offerte deducibili per il sostentamento del clero", dei redditi propri dei presbiteri, di una quota fissa delle offerte fatte direttamente in parrocchia e dei ricavi provenienti dalla gestione degli istituti diocesani per il sostentamento del clero. Il doppio canale si giustificava con lo scopo di mantenere diffusa e consapevole tra clero e laici la responsabilità rispetto alla dimensione economica della vita delle organizzazioni ecclesiastiche, espressa più dalle seconde quattro modalità che dalla prima. Negli anni, però, al boom del gettito dall'"otto per mille" ha corrisposto un decremento continuo del contributo proveniente dagli altri canali. Nell'abbondanza delle risorse disponibili ogni proposta di rilancio delle altre forme di esercizio del "dovere di contribuire alle necessità economiche della Chiesa" (come insegna il catechismo) si è arenata. A oggi, e per quanto riguarda il sistema di sostentamento nato dal nuovo Concordato (cui certo non si riduce il finanziamento della Chiesa), per la propria sussistenza economica le organizzazioni ecclesiastiche cattoliche si affidano più alla identificazione che alla partecipazione, si affidano più a quanto può essere coltivato dal centro e mediaticamente, che a quanto dipende dalla capacità diffusa di generare partecipazione. 
diocesi) cresce di oltre il $350 \%$ tra il 1990 e il 2010. Nello stesso periodo, la quota di risorse provenienti dall' "otto per mille" e destinate agli organismi centrali della CEI cresce in misura più che proporzionale. Se, come si è appena visto, tra il $1990 \mathrm{e}$ il 2010 i fondi in qualche modo destinati alle diocesi erano cresciuti del $350 \%$, quelli assegnati agli organismi centrali della CEI crescono del $1.151 \%$ (più del triplo).

Nello stesso periodo, aumenta la forza di quel centro e vertice costituito dagli organismi centrali della CEI e diminuisce l'intraprendenza del clero (in passato capace di generare opere anche di elevato profilo economico) in periferia; contemporaneamente non decolla l'impegno nel promuovere l'esercizio della responsabilità dei fedeli a sovvenire e anche a controllare la vita economica della Chiesa. In sintesi: mentre sino agli inizi degli anni ' 80 il sistema di sostentamento del clero (porzione decisiva della dimensione economica della vita ecclesiale) si caratterizzava (Consorti 2010, p. 130ss.) per un mix di forte dipendenza dalla decisione di una autorità extra-ecclesiastica (determinante l'ammontare dei trasferimenti) e assenza di una autorità religiosa centrale capace di regolare la distribuzione degli stessi, con il nuovo regime si realizza sia una fortissima riduzione della dipendenza dalla autorità extra-religiosa che una forte centralizzazione attraverso la formazione di una autorità religiosa che presiede alle decisioni in questo ambito. Il problema che si apre è così quello del contrasto tra il valore in linea di principio attribuito dalla costituzione ecclesiastica cattolica ${ }^{6}$ alle diocesi o chiese particolari, enormemente maggiore rispetto a quello attribuito alle istanze di cooperazione ecclesiale (soprattutto a quelle di livello nazionale!), e la redistribuzione reale di potere intervenuta tra diocesi e vertici CEI attraverso questa particolare interpretazione pratica del nuovo regime di sostentamento. Interpretazione che non era certamente l'unica possibile (cfr. ad es. nota n.6).

Per valutare correttamente le trasformazioni appena richiamate occorre evitare alcuni luoghi comuni, privi di qualsiasi fondamento. (i) Le nuove forme del sovvenire alle necessità economiche della Chiesa, che non sostituiscono le altre (salvo il vecchio sistema beneficiale) ma si affiancano loro, costituiscono in termini istituzionali un enorme passo in avanti sia sul piano civile sia su quello ecclesiale, come una consistente letteratura (tanto giuridica quanto teologica) ha messo in chiaro. Anche su scala internazionale esse offrono una delle più qualificate espressioni del combinarsi di una doppia opzione per il modello della libertà religiosa: quella operata dalla Costituzioni Italiana del 1948 (cfr. ad es. art.8) e quella operata dal magistero con il Vaticano II. (ii) Il flusso economico generato dall'"otto per mille" (e dalle offerte deducibili per il sostentamento del clero) è sottoposto a un livello di revisione e di controllo (tra l'altro anche bilaterale, ovvero con il coinvolgimento dell'amministrazione statale) che, ovviamente sempre perfettibile, ha pochi analoghi non solo in ambito religioso, ma anche in altri ambiti sociali (politici inclusi). Infine, i possibili impieghi del flusso

6 Cfr. Lumen gentium, in part. capp.1-3. 
finanziario generato dall'"otto per mille" sono definiti per accordi tra le parti aventi valore di legge. Nella pratica, poi, (iii) il nuovo volume di potere dei vertici della CEI, generato dalla possibilità di curare l'amministrazione di questi fondi, è stato spesso impiegato per indurre comportamenti virtuosi e per sostenere elementi di modernizzazione nella vita delle diocesi: come ad es. quando si è deciso di far dipendere il trasferimento dei fondi alle stesse diocesi dall'adozione da parte di queste di forme di pubblicità dei bilanci prima raramente praticate. (iv) Tutte le politiche di raccolta e di spesa adottate dal 1988 in sede CEI, a partire dai bilanci annuali, hanno ricevuto l'approvazione della intera assemblea dei vescovi italiani. In sintesi, solo una volta chiariti i caratteri del sistema e la sua elevata qualità civile ed ecclesiale, è possibile portare l'attenzione sull'interpretazione pratica che di questo sistema è stata effettivamente data: interpretazione con forti tratti di centralizzazione e di verticalizzazione, senz'altro legittima, ma non l'unica possibile.

Il processo di verticalizzazione e centralizzazione appena descritto, come ogni processo sociale non è mai la pura e semplice applicazione di un progetto. Tuttavia in alcuni casi avviene che, magari solo per brevi periodi, il processo reale sia concepito e governato da un'organizzazione più sistematicamente di quanto non si verifica nella maggior parte dei casi. In questo senso - pur sempre con la dovuta prudenza - per il processo sin qui descritto si può sensatamente impiegare la categoria di "progetto". Non si possono cioè considerare del tutto casuali gli effetti di centralizzazione e di verticalizzazione della struttura dell'autorità religiosa cattolica verificatesi in Italia e che sono stati appena indicati. Effetti - vale la pena ripeterlo - che devono essere riconosciuti anche come originalissimi, come ecclesialmente inediti, perché localizzati a un livello - quello nazionale e per di più italiano - in linea di principio di modestissima importanza per la vita e l'autocoscienza cattolica, oltre che di recentissima formalizzazione. E all'esplicita formulazione di un "progetto" somiglia molto quanto il Card. Camillo Ruini dichiarò in una delle sue prime uscite pubbliche da Presidente della CEI, in occasione del Consiglio Permanente della stessa l'11 marzo 1991. In quell'intervento si poneva in chiaro che lo sviluppo della CEI avrebbe dovuto essere risposta alla "necessità di una presenza pubblica della Chiesa in Italia che abbia una vera e adeguata dimensione nazionale, ruolo che in via principale, anche se certamente non esclusivo, può essere esercitato soltanto dal corpo dei vescovi e quindi, nella pratica quotidiana, attraverso lo strumento della Conferenza episcopale" (Acerbi 2006, p. 84). 


\subsection{Le parrocchie}

Lo stesso processo di semplificazione o se si vuole di riduzione della capillarità della base della struttura religiosa del cattolicesimo italiano si riflette anche a livello di parrocchie. Tra il 1976 ed il 2011 le parrocchie italiane passano da 28.379 a 25.503 [A], diminuendo dunque di un po' più del $10 \%$.

Se però consideriamo le sole parrocchie con un parroco (non importa se in esclusiva o condiviso con altre parrocchie, non importa se appartenente al clero diocesano od a quello religioso), il saldo negativo diviene ben più marcato. Queste passano da 25.067 a 20.742, con un calo non del $10 \%$, ma del $17 \%$ [A].

\section{Il personale}

Per tutti i tipi di organizzazioni quelle umane sono alcune delle risorse più preziose (Luhmann 2005, p. 183ss., 230ss.). Per le organizzazioni religiose, come per tutte le organizzazioni professionali ciò vale in modo ancor più stringente (Abbott 1988).

Un'adeguata valutazione delle cifre che seguono può trarre vantaggio dal ricordare che nei quaranta anni considerati la popolazione residente in Italia cresce di poco meno del $20 \%$, passando da poco più di 50 milioni a poco più di 60 milioni di residenti. ${ }^{7}$

\subsection{II clero diocesano}

È il caso di partire dalla componente costituita dal clero diocesano, sia per il grado di controllo che esso ordinariamente esercita sulla struttura della autorità religiosa cattolica sia per la dimensione che questa componente del clero raggiunge nel corso del Novecento rispetto alla dimensione cui si riduce la componente di clero appartenente a ordini religiosi (Brunetta 1991, p. 436). Nel 1970 erano presenti in Italia e in servizio per le diocesi italiane 42.868 preti diocesani. Nel 2011 il loro numero è di 32.475 , il che equivale alla diminuzione di un quarto in quaranta anni $[\mathrm{A}]$. Una seconda fonte $\left[\mathrm{G}^{8}\right]$, che si basa su criteri leggermente diversi e su diverse procedure di raccolta dei dati, fornisce valori un poco più ridotti (29.646 preti diocesani a fine 2012), ma nella sostanza conferma la ten-

7 La nostra analisi, per ragioni evidenti, dovrà restringersi al personale ecclesiastico attivo sul territorio italiano, ignorando il fatto che il cattolicesimo italiano nel corso del Novecento ha fornito un grande contributo alla proiezione missionaria della Chiesa, e che questo contributo ha fatto invece segnare un significativo declino negli ultimi decenni del XX secolo e nel primo del XXI.

8 Per diverse ragioni probabilmente più affidabile. 
denza generale per i venti anni per i quali quest'ultima fonte è in grado di produrre informazioni.

Questa già importante contrazione numerica, tuttavia, non è in grado di rappresentare da sola per intero i problemi incontrati dal reclutamento di personale per il clero diocesano. Nella composizione di questa popolazione, infatti, sono intervenute anche altre trasformazioni assai rilevanti, ma non bene espresse dal pur macroscopico calo assoluto.

Per effetto del combinarsi di tre principali processi - la diminuzione delle ordinazioni, pur con andamento non lineare (Molina 2005. P. 65; [A]), l'aumento dell'età media all'ordinazione [G], un aumento della speranza di vita nel clero maggiore di quella fatta registrare dalla popolazione maschile italiana - i preti diocesani del 2011 non sono solo in numero inferiore rispetto ai loro colleghi del 1970 , ma sono anche mediamente più anziani. Solo negli ultimi dieci anni (per il decennio 2003-2012 si dispone di studi più precisi [G]) l'età media dei maschi italiani (con più di 24 anni) è passata da 49,5 anni a 51,4, mentre quella dei preti diocesani nati in Italia è passata da 60,3 anni a 61,5. A fine 2012 tra i preti diocesani nati in Italia il $16 \%$ aveva più di 80 anni, erano il 12,5\% nel 2002 e solo il 5,4 \% nel $1991[\mathrm{G}]$.

In aggiunta a ciò, diversi altri indicatori segnalano un allentamento dei processi di formazione e di selezione delle nuove leve di clero diocesano. (i) Negli ultimi anni cresce il rapporto tra ordinati e seminaristi, mentre diminuisce la quota di seminaristi maggiori che abbandona il percorso formativo al ministero. Entrambi i fenomeni si verificano nel quadro di un calo degli ingressi in seminario. In pratica, chi oggi chiede di entrare in seminario ha molte più probabilità di diventare prete rispetto a quelle che aveva un suo collega di alcuni decenni orsono [A] . (Senza contare anche un certa tendenza da parte dei candidati ai quali non si consente di continuare il percorso di formazione presbiterale a rivolgersi ad altri seminari, e con successo.) (ii) La fortissima riduzione della quota di ordinati che ha cominciato il proprio iter nel seminario minore, ${ }^{9}$ comporta l'arrivo all'ordinazione di individui sui quali è stata esercitata una azione formativa minore (sotto il profilo culturale come sotto quello umano) per durata - certamente - e probabilmente - anche per intensità e selettività. Entrano dunque nel ministero elementi tra loro molto meno omogenei di quanto verificatosi in questi ultimi tre o quattro secoli, nonostante la loro omogeneità resti un requisito largamente presupposto dalle modalità di funzionamento e di gestione delle organizzazioni ecclesiastiche che impiegano clero diocesano. (iii) L'innalzamento dell'età media

9 Nel 2005, entro un campione rappresentativo del clero diocesano italiano (Bressan 2006), solo il $54 \%$ dichiarava di aver frequentato in seminario le scuole medie superiore. La stessa percentuale era del $64 \%$ tra gli ordinati tra il 1954 ed il 1968, del $61 \%$ tra gli ordinati tra il '69 e l' 86 , del $36 \%$ tra gli ordinati tra il 1987 ed il 2003. In un campione casuale di oltre 1000 seminaristi la stessa percentuale scendeva ancora, attestandosi al $23 \%$. 
all'ordinazione porta nel ministero individui che hanno nel proprio bagaglio biografico le più diverse esperienze umane e sui quali una azione formativa, già quantitativamente ridotta, produce inevitabilmente effetti ancora meno profondi. ${ }^{10}$ Senza supporre in alcun modo che questo costituisca necessariamente una trasformazione di segno negativo, ma solo che si tratti obiettivamente di una grande diversità rispetto al passato, va detto che anche nel clero diocesano italiano ha fatto la sua comparsa quello che in letteratura è noto come "clero alla seconda carriera" (second career clergy, Nesbitt 2007). L'età media all'ordinazione era per i preti diocesani di 26 anni nel 1970; era cresciuta di meno di due anni rispetto a trenta anni prima (1940/1970), mentre era destinata a crescere di 4 anni nel trentennio successivo (1970/2003), e poi ancora successivamente sino a toccare a livello nazionale i 32 anni nel 2012 (Molina 2005, p. 43 e [G] ${ }^{11}$ ). (iv) Infine, sopra a tutte le altre pratiche di reclutamento non convenzionale poste in essere dalle diocesi,${ }^{12}$ e certamente per qualche verso stimolata e facilitata dall'arrivo del primo Papa straniero, va considerato che nel corso del periodo preso in considerazione si è andata diffondendo la pratica del ricorso a clero straniero, prevalentemente proveniente da aree tra le quali alcuni paesi dell'Est Europa, l'Africa, alcune zone del Sud America e dell'Asia. (Tra il 1983 ed il 2012 entrano nel clero diocesano attivo in Italia circa 3.700 presbiteri provenienti dall'estero: 1.426 come seminaristi che ricevono l'ordinazione in Italia e 2.280 come presbiteri già ordinati che si escardinano da diocesi estere per incardinarsi in diocesi italiane.) Ancora una volta siamo in grado di seguire con una certa precisione questo fenomeno solo a partire dal $1990[\mathrm{G}]$ ed è possibile dire che in quel momento già poco meno del $5 \%$ dei 31.492 preti diocesani incardinati in diocesi italiane era di origine straniera (secondo stime per difetto quella percentuale era solo del $2 \%$ nel 1971). A fine 2012 lo stesso valore era salito a poco meno dell'7 \% dei 29.646 preti diocesani in servizio per le diocesi italiane. Se per un verso il fenomeno ha dimensioni inferiori a quelle raggiunte dalla presenza di stranieri nella popolazione residente in Italia, la sua valutazione richiede alcune attenzioni. Mentre è ancora rara la presenza di stranieri tra i professionisti in Italia, essa risulta assai diffusa tra gli individui con occupazioni largamente percepite come meno qualificate. Il risultato è che lo scarso interesse dei nativi per quelle professioni finisce per fa-

10 Dall'indagine del 2005 appena citata (Bressan 2006) risulta che hanno frequentato l'università prima di entrare in seminario il $5 \%$ dei presbiteri ordinati prima del ' 68 , il 10 di quelli ordinati fino al 1986, il $28 \%$ di quelli ordinati fino al 2003, e addirittura il $36 \%$ dei seminaristi.

11 Per altro con un andamento del tutto parallelo a quello dell'età media al primo matrimonio, per uomini e donne, e a quello dell'età media al primo figlio per le donne. 12 Dei quasi 30.000 presbiteri diocesani in servizio nelle diocesi italiane a fine 2012, circa il $13 \%$ aveva cambiato diocesi d'incardinazione dal momento dell'ordinazione o prestava servizio in una diocesi diversa da quella nella quale risulta incardinato. In entrambi i casi si tratta di fenomeni che rivelano una certa instabilità dei presbiteri diocesani. 
cilitare questo esito e poi un processo che almeno in parte si autoalimenta. Nell'importazione di clero straniero una componente di rischio è evidente. In proposito vale ancora la pena notare che il ricorso a clero di origine non locale non è una politica diffusa in modo omogeneo tra le regioni, ma ha tratti simili ovunque essa venga praticata. Infine, e questo è l'elemento i cui effetti si proiettano più profondamente verso il futuro della struttura religiosa del cattolicesimo italiano, si ricorre a clero straniero innanzitutto per affrontare la carenza di giovane clero di origini italiane. Al momento, in alcune aree del paese, il clero sotto i 40 anni è di origine straniera per quasi il $50 \%$ e, comunque, a fronte di una età media del clero diocesano nato in Italia che a fine 2012 era pari a 61,5 anni, quella del clero nato all'estero alla stessa data era di 46,7 anni. A questo dato si aggiunga anche che le informazioni ufficiali per un verso sottostimano il fenomeno (non potendo considerare presenze informali, come quelli di clero straniero impegnato per gli studi ecclesiastici in facoltà romane o del resto d'Italia), mentre non rendono ancora percepibile un lieve rallentamento del ricorso al clero straniero che sembra in atto dal 2010 [G].

Insomma, dopo quaranta anni le diocesi italiane possono contare su meno preti diocesani, su preti diocesani mediamente più anziani, nonché su preti diocesani passati per una formazione meno intensa e forse anche (ma questo è più difficile da stabilirsi con certezza) attraverso una selezione meno severa. Anche nel caso del clero, insomma, si verifica in un modo o nell'altro quello che si sta verificando per le élites in pressoché tutti i settori della società italiana.

\subsection{I religiosi e le religiose}

Purtroppo solo pochi dati sono a disposizione con riferimento a una delle componenti più importanti e più caratteristiche del cattolicesimo religioso: la vita religiosa - maschile e femminile - con i suoi ordini, le sue congregazioni, le sue varie e molteplici forme di organizzazione. ${ }^{13}$

Per quanto riguarda l'Italia e il periodo che stiamo considerando si può dire che i presbiteri religiosi presenti nelle diocesi passano da 22.423 (nel 1970) a 15.202 (nel 2012), perdendo dunque circa un terzo della propria consistenza e subendo un calo molto più forte di quello conosciuto dal clero diocesano. Se ci sono degli indizi per ritenere probabile che nel calo quantitativo non si esprima che una parte delle trasformazioni sperimentata da questo settore del personale ecclesiastico, non ci sono però informazioni empiriche in grado di documentarle con precisione.

13 Queste note puramente quantitative da un lato certo non esauriscono il quadro dei cambiamenti che interessano la vita religiosa (Wittberg 1994; Finke Wittberg 2000) e dall'altro, con riferimento al caso italiano, ben si inseriscono nel quadro elaborato da autori come Dal Piaz (2013; cfr. tra l'altro anche Borghesi / Castegnaro / Dal Piaz / De Sandre 2007). 
Tra le poche informazioni affidabili vi è quella per cui i presbiteri religiosi inseriti nel sistema di sostentamento del clero gestito dalla CEI (in ragione degli impegni pastorali a livello diocesano o parrocchiale di quegli individui) l'età media sale lievemente dai 55 anni ai 55,8, ma per effetto della crescita di quella dei nati in Italia da 55,4 a 59,2 e del calo di coloro che non sono nati in Italia da 46,9 a 44,56 [G]. Sempre tra i presbiteri religiosi inseriti nel sistema di sostentamento del clero della CEI la quota di nati all'estero passa dal $4,5 \%$ al $23 \%$.

In Italia, dal 1976 al 2011 le religiose professe (quelle che hanno pronunciato voti definitivi o equivalenti) sono passate 145.090 a 89.264 [A]. In quaranta anni la perdita è dunque di circa 56.000 unità, per effetto di questa le religiose presenti oggi in Italia sono pari al 61,5\% di quelle presenti nel 1976. Si tratta evidentemente di una contrazione molto più consistente di quella fatta segnare dai religiosi, e ancor più marcata rispetto a quella subita dal clero diocesano.

È poi molto probabile che questo saldo nasconda - come nel caso del clero diocesano e dei religiosi - trasformazioni anagrafiche e di provenienza che al momento non possono però essere quantificate. Così come è molto probabile che in non tutti i settori della vita religiosa femminile il saldo negativo abbia le stesse dimensioni. Anche in questo caso è ragionevole ipotizzare che nelle forme di "vita attiva" esso sia superiore per lo meno rispetto ad alcune esperienze di vita contemplativa e di clausura.

Quello che però è possibile documentare, sebbene attraverso numeri piuttosto piccoli [A], è che la vita religiosa femminile resta ancora, almeno un poco, spazio di ricerca e di sperimentazione di nuove vie. Il calo degli ultimi trenta anni sembra, infatti, dover essere attribuito per intero a organismi di diritto pontificio (ordini e congregazioni tradizionali), mentre le realtà di diritto diocesano (spesso le realtà più recenti, più flessibili e anche con maggior ricambio) presentano nel complesso una tenuta (e anzi un lievissimo incremento).

\subsection{Altri attori}

Una comparazione tra le dimensioni del personale e le forme della struttura dell'autorità religiosa cattolica in Italia intorno al 2010 e le une e le altre intorno al 1970 fa emergere una serie di $d e$-formazioni (termine - ricordiamolo - esente da ogni connotazione valutava!) che non possono essere spiegate solo con quanto ricordato sin qui. Sul mutamento rappresentato, infatti, hanno influito anche cambiamenti intervenuti nell'estensione e nelle forme della partecipazione laicale alla vita delle istituzioni ecclesiastiche. Essa ha dovuto fare i conti anche con il nuovo protagonismo assunto nell'ambito del cattolicesimo religioso da organizzazioni religiose non controllate dall'episcopato e molto diverse dalle tradizionali, e ancor ben vive, associazioni ecclesiali del laicato cattolico. Com'è ben noto, il fenomeno non si è verificato solo in Italia, e anzi altrove si è manifestato in modo ancor più evidente che in Italia (da Wuthnow 1988 a Chaves 2004, 2006, 
2011, a Turner 2012). Nel caso del cattolicesimo, e particolarmente di quello italiano, l'originalità sta nei rapporti che si negoziano (a vari livelli) tra molte di queste nuove realtà e l'autorità religiosa.

Nonostante alcuni "nuovi movimenti" cerchino di egemonizzare l'immagine e la rappresentanza di questo complesso fenomeno di diversificazione dell'offerta religiosa cattolica, essi non solo non rappresentano la totalità o i caratteri generali di questo processo di diversificazione, ma neppure la parte quantitativamente più consistente. Semmai ne costituiscono la porzione con maggiore capacità di autorappresentazione, intra ed extra religiosa, e ciò - paradosso solo apparente proprio grazie a un maggiore livello di organizzazione e di centralizzazione. In realtà, dello stesso processo di diversificazione interna dell'offerta religiosa cattolica sono parte integrante e quantitativamente prevalente nuove o profondamente rinnovate forme di religiosità popolare, nuove esperienze di monachesimo (alcune delle quali già ben strutturatesi e influenti) e, soprattutto, una diffusissima platea di gruppi religiosi di dimensioni anche modeste e spesso privi di etichetta e di legami con altre realtà. $\grave{E}$ in questi termini, e senza raggiungere neppure gli apici che tocca in altri contesti nazionali (come nell'area di lingua spagnola e portoghese), che il processo di diversificazione dell'offerta e degli stili di consumo religioso al quale ci stiamo riferendo si manifesta anche in Italia.

Se si vuole comprendere questo fenomeno, è sconsigliabile scegliere la quantità come chiave interpretativa primaria. Infatti, la tesi molto diffusa di una sostituzione dell'associazionismo laicale tradizionale - in primis quello dell'Azione Cattolica Italiana (ACI) - con i "nuovi movimenti" non è confortata da elementi empirici. L'ACI conserva tuttora le dimensioni quantitative più consistenti. Dichiara infatti di parteciparvi [E] circa il $2 \%$ della popolazione italiana adulta, una quota superiore a quella raggiunta dalla somma delle indicazioni raccolte da tutti gli altri principali "nuovi movimenti" religiosi cattolici. Ancora, con riferimento al 2011, l'ACI conta una presenza formalizzata in circa 6000 parrocchie italiane (su oltre 25.000, [C]), il Cammino Neocatecumentale in circa $1100 .{ }^{14}$ Semmai, stabilmente più diffusa dell'ACI appare la miriade di gruppi parrocchiali senza etichetta.

Invece di un fenomeno di sostituzione dell'associazionismo con i nuovi movimenti, si dovrebbe parlare di $(i)$ generale contrazione della partecipazione della popolazione ad aggregazioni religiose di qualsiasi tipo, di (ii) notevole turn over e di una diffusissima improvvisazione ed estemporaneità educativa in questa partecipazione, e di (iii) una enorme diversificazione di questo fronte con un impatto notevole sulla effettiva qualità ecclesiale e civile dello stesso. Non a caso nel 1991 la CEI dovette pubblicare una Nota e poi, alla metà degli anni 2000, ribadirne alcuni contenuti in diversi documenti.

14 Dato elaborato dal Cammino Neocatecumenale a livello nazionale a partire da informazioni raccolte, a titolo meramente indicativo, dalle équipe dei catechisti delle zone. 
I nuovi movimenti religiosi cattolici, nelle forme più diverse, tendono a offrire alternative rispetto alle normali prassi catechetiche, liturgiche, spirituali, sino a ritagliarsi di fatto uno spazio nei processi di formazione e di selezione del clero diocesano (giungendo sino alla istituzione e gestione di seminari separati per candidati al presbiterato provenienti da qualche particolare esperienza di movimento). Se potesse prendere atto della loro presenza, Max Weber probabilmente si chiederebbe se, nell'ambito del cattolicesimo religioso italiano, esiste ancora un "monopolio della coercizione ierocratica legittima", ovvero se questo ha ancora forma ecclesiale.

In termini evolutivi non va commesso l'errore di interpretare i protagonisti e i risultati del processo di diversificazione e di frammentazione dell'offerta religiosa come frutti del Vaticano II (1962-1965). In questa sede è sufficiente ricordare che il manifestarsi di queste nuove organizzazioni religiose non ecclesiastiche (piccole e grandi) si verifica in periodi poco o spesso molto precedenti alla celebrazione del Concilio Ecumenico Vaticano II. ${ }^{15}$ La sua diffusione, non la sua nascita, va connessa alla crisi nella ricezione del Vaticano II e, a volte, alla almeno parziale sfiducia nel suo magistero. Questa dato ci riporta, semmai ce ne fossimo distaccati, al filo principale del ragionamento.

$\grave{E}$ il cattolicesimo religioso italiano in generale che si rafforza proprio in relazione alla crisi e alle incertezze nella ricezione del magistero del Vaticano II. In questo processo, per un verso ciascuno dei suoi "frammenti", dal movimento più consistente al gruppo parrocchiale più piccolo, tende a sottrarsi al controllo e alla regolazione del vertice della struttura della autorità religiosa. Per altro verso, diversamente da quello che si è verificato nell'ambito del protestantesimo nord e sudamericano, questi frammenti non escono definitivamente dal campo religioso cattolico, non coniano un nuovo brand e una nuova identità. Essi non fanno mancare la legittimazione simbolica al pontefice (più critica è la relazione con $\mathrm{i}$ singoli vescovi diocesani), né la cooperazione a singole iniziative, ma in genere le rinegoziano in cambio di una certa dose di altri vantaggi a partire da quello minimo di una certa dose di visibilità. In una situazione come quella italiana, dove la dominanza del cattolicesimo sul campo religioso ha da tempo assunto forme di larga flessibilità, per ciascuno di questi nuovi imprenditori religiosi cattolici postecclesiali "uscire", adottare un brand proprio e diverso, sarebbe allo stesso tempo molto costoso (implicherebbe sostenere un conflitto con un gigante e far lievitare per i consumatori religiosi il prezzo dei propri beni e servizi religiosi) e poco redditizio (anche perché il controllo religioso che l'autorità di questo gigante esercita non è poi così asfissiante). Per questa miriade di piccoli e grandi frammenti è più conveniente adottare una diversa strategia. Dopo essersi sottratti a una presa già modesta, ricontrattano il proprio contributo in termini di partecipazione e di legittimazione simbolica, spesso ottenendo abbondanti eccezioni ed esenzioni (quali neppure gli ordini religiosi più forti avevano ottenuto, cfr. Prodi

15 Per un quadro dei principali protagonisti del fenomeno cfr. Favale e coll. 1991. 
2010, p. 216ss.). Interessantissime, sotto questo profilo, sono vicende come quelle che hanno caratterizzato la discussione degli statuti e delle prassi di tanti soggetti, dall'Opus Dei a Comunione e liberazione, al Cammino Neocatecumenale e così via. (Si potrebbe parlare forse di una "prelaturizzazione" del cattolicesimo, con riferimento alla diffusione effettiva e più ancora potenziale dell'istituto, molto discusso, della prelatura personale?)

È su questo sfondo che diventa comprensibile la crisi cui deve far fronte il modello canonico di vescovo e di prete, l'Azione Cattolica Italiana, i religiosi e le religiose "non autocefali", i portatori di ruoli come quello di catechista e di teologo. In termini assoluti e ancor di più in termini comparativi, essi non sono (o per lo meno non sono ancora) in numero esiguo o insufficiente. Al contrario. Essi tuttavia rappresentano elementi di un cattolicesimo religioso di tipo ecclesiale che ormai - almeno dai primi tempi del pontificato di Giovanni Paolo II - appare apertamente messo in crisi anche dall'interno. Spesso, poi, questi attori hanno anche introiettato questa crisi, riconducendola a ragioni quantitative, ingigantite nel loro pur reale peso. Il risultato è quello di assumere troppo spesso una posizione difensiva o addirittura passiva. Tutte le espressioni della Chiesa del Vaticano II e in qualche modo "montiniana" sono oggi in difficoltà, strette tra un ambiente esterno ancora più difficile del previsto e un ambiente interno a sua volta diviso tra nostalgie improbabili e strategie di frammentazione a volte anche vincenti, ma non facilmente compatibili con il mantenimento di una forma ecclesiale di cattolicesimo.

Nel frattempo, quanto va consolidandosi - inedito e interessantissimo - è il formarsi di un nuovo tipo di struttura della autorità religiosa cattolica, altrove definita "multi channels": fatto non più di un canale, ma di molti e tra loro diversi canali (Diotallevi 2001, p. 104ss.). I canali di questa struttura dell'autorità religiosa, le "vie di comunicazione" (cfr. Luhmann 2005, p. 185 e poi inevitabilmente i loro programmi), non solo si moltiplicano e per tanti versi divengono paralleli e non comunicanti, ma si diversificano per tipologia. In alcuni casi diventano addirittura estremamente informali. Ciò implica, al vertice, una netta perdita della distinzione tra carisma e autorità, e dunque la messa in crisi di uno dei tratti distintivi della istituzione religiosa di tipo ecclesiale. Ovvero di quella forma sociale (ben più che solo religiosa) che il cattolicesimo, forse più di qualsiasi altra tradizione cristiana, aveva tenacemente cercato di conservare. Più il cattolicesimo si dà un futuro da sola religione, e da religione nella forma attualmente dominante (Turner 2011, xii), più al suo interno faranno fatica le istituzioni dell'ecclesialità.

Sarà innanzitutto (anche se non esclusivamente) con riferimento a queste deformazioni - in quanto trasformazioni che prendono le distanze dalla formachiesa - che si potrà parlare di "neoclericalismo". Quello che va formandosi lungo questa rotta - già intrapresa anche se non ancora irreversibile (Diotallevi 2014b, 2014a) - è un clericalismo appunto di tipo nuovo. È fatto più di esposizione e di visibilità che di volontà e possibilità di effettivo governo (sia intra che extra 
religioso). È un clericalismo con più risorse (di vario tipo) e nello stesso tempo-in fondo - con meno politiche e con progetti di raggio più corto.

In definitiva, il processo di centralizzazione e di verticalizzazione della struttura dell'autorità religiosa cattolica in Italia, che sconta l'assottigliarsi per lo meno quantitativo del personale a disposizione, una volta che si combina con le tre $d e$ formazioni appena descritte, appare in un'immagine più realistica. Esso in parte si conferma, in parte si depotenzia, in parte funge da bilanciamento di quelle stesse tendenze $d e$-formanti. Il costo complessivo di questo fascio di tensioni è il ridursi (non l'annullarsi) del raggio di influenza della tradizione cattolica oltre il perimetro del religioso. Non a caso, questi quaranta anni sono quelli della centralizzazione dell'autorità religiosa cattolica in Italia e gli anni del dominio della "pastorale" e delle "pastorali" su tutte le altre forme di apostolato nella Chiesa. Causa ed effetto di ciò, essi sono stati inevitabilmente gli anni della crisi del cattolicesimo politico, della declinante rilevanza del cattolicesimo nel mondo economico (sindacale, imprenditoriale, finanziario, ecc.), nel mondo della scienza e della cultura. Contemporaneamente sono stati gli anni del declino della correlazione tra le varie dimensioni della religiosità individuale: partecipazione, identificazione, conoscenza, credenza, esperienza. Per questo, ci sembra che alla fine abbia senso parlare proprio di "neoclericalismo debole". Il progetto elaborato almeno per l'Italia in tanti settori di vertice dell'apparato ecclesiastico durante i pontificati di Pio XI e Pio XII, e preparato durante i decenni precedenti (Moro 1988), era quello di una società tradizionale governata con metodi moderni, fatti funzionare da un laicato esecutore, governato dal clero, governato a sua volta da un episcopato operativo con poteri quasi prefettizi, conferiti dal Romano pontefice. Questo, sì, era il disegno o solo il sogno di un "vero clericalismo"; non quello che si accontenta di qualche passaggio televisivo, di un po' di risorse per le public relations e di qualche meeting religioso.

\section{Un confronto internazionale}

È difficile valutare trasformazioni come quelle appena documentate senza ricorrere anche ad un confronto internazionale. In questo però, come in molti altri casi, una operazione del genere si presenta ancora più difficile ${ }^{16}$. In una sede come

16 Il primo problema è costituito dalla scarsità di dati dotati di tutte le caratteristiche che ne rendano possibile la comparazione. A questo si somma la diversità delle situazioni di partenza, delle dinamiche in atto in un determinato momento in ciascuno dei casi comparati, nonché dei contesti e dei fattori all'opera anche per tutto il periodo considerato. Per far solo un esempio, si pensi a quanto incide la storia ecclesiastica secolare o la orografia dei diversi territori nella definizione del tessuto parrocchiale. Notevolissima è l'escursione della dimensione demografica media della parrocchia anche tra paesi come Spagna, Francia e Italia (Diotallevi 2010b). 
questa è consigliabile limitare il confronto a pochi indicatori ed ai valori che essi assumono nei paesi il cui panorama religioso ha almeno un paio di importanti tratti comuni: l'appartenenza all'area dell'Europa centro-occidentale e la posizione almeno tradizionalmente dominante del cattolicesimo in ambito religioso. Questa scelta spinge in sostanza a prendere in considerazione un paese di area tedesca, l'Austria, due di area francofona, la Francia ed il Belgio, due iberici, la Spagna ed il Portogallo, un paese di area britannica, l'Irlanda, e dell'Italia (inclusi i valori relativi al territorio della Repubblica di San Marino e della Città del Vaticano) (tab.1).

Come si vede facilmente, in tutti i casi [A] si tratta di paesi che hanno visto crescere (seppur con notevoli differenze) la propria popolazione complessiva nel corso dei quarant'anni considerati, e anche i "cattolici"17 - in generale questi ultimi con ritmi appena un poco inferiori a quelli dei residenti, ma con eccezioni marcate e di segno negativo nel caso della Francia, del Belgio e dell'Austria.

Per quanto riguarda la forma della struttura dell'autorità religiosa sono due, ma assai importanti e tra loro diverse, le eccezioni che si possono osservare. Rispetto a un andamento generale, tra il 1976 e il 2010 cresce lievemente il numero delle diocesi e quello delle parrocchie. Le due eccezioni sono quella francese e quella italiana. Nel primo caso si è assistito a una sostanziale tenuta del numero delle diocesi $(+2)^{18}$ e addirittura a una riduzione delle parrocchie pari al $60 \%$ (da 38.236 a 15.765). Nel secondo caso, quello che più direttamente ci riguarda, le diocesi sono state ridotte del $20 \%$ e le parrocchie del $10 \%$. Il dato generale e le due eccezioni appena ricordate fanno poi da sfondo a un'informazione forse ancora più importante. Con l'eccezione del Portogallo, aumentano sensibilmente le parrocchie senza parroco residente (in Italia e Spagna però meno che altrove), mentre calano drasticamente in Francia anche in virtù del taglio drastico al numero delle parrocchie cui si è appena fatto cenno.

In termini di personale la valutazione dei dati a disposizione si fa ancora più difficile, essendo praticamente impossibile stimare per gli altri paesi il valore di variabili cruciali come l'età media del clero o la presenza di clero straniero. Entro tali limiti è però possibile identificare quattro fenomeni. (i) Il clero diocesano cala ovunque. In Italia il calo è più contenuto, arrestandosi a una perdita del $20 \%$, mentre raggiunge i livelli più elevati in Belgio e in Francia dove il clero diocesano presente nel 2010 è di poco superiore per numero al $40 \%$ del clero diocesano presente nel 1976. (ii) La forbice si allarga ulteriormente nel caso dei seminaristi maggiori per il clero diocesano. Tra il 1976 e il 2010 in Italia si registra addirittura un lievissimo aumento $(+2 \%)$, mentre negli altri paesi sopravvive un numero di seminaristi che nel migliore dei casi (Portogallo) è il $70 \%$ e nel peggiore (Belgio) è il $22 \%$ dello stesso valore nel 1976. (iii) Una dinamica analoga alle precedenti, anche se ancor più marcatamente negativa e più generale, concerne le religiose.

17 In questo caso sinonimo di battezzati nella Chiesa cattolica.

18 Le quali però erano state fortemente ridotte non molto tempo prima del 1976. 


\begin{tabular}{|c|c|c|c|c|c|c|c|c|c|}
\hline & 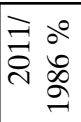 & $n$ & $n$ & ले & $i$ & 3 & 6 & $\overrightarrow{6}$ & $n$ \\
\hline & 을 & 守 & $\begin{array}{l}\stackrel{\leftrightarrow}{\Omega} \\
\text { ă }\end{array}$ & $\begin{array}{l}0 \\
\text { Dे } \\
\dot{+}\end{array}$ & $\stackrel{n}{n}$ & 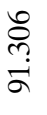 & 勇 & $\begin{array}{l}\stackrel{q}{*} \\
\stackrel{+}{+}\end{array}$ & 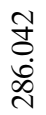 \\
\hline 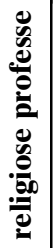 & $\frac{2}{2}$ & $\begin{array}{l}\underset{\forall}{J} \\
\underset{J}{J}\end{array}$ & 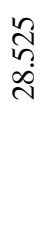 & $\begin{array}{l}\stackrel{\infty}{0} \\
\stackrel{0}{\circ} \\
\stackrel{8}{\circ}\end{array}$ & $\begin{array}{l}\stackrel{\sigma}{ٍ} \\
\stackrel{\leftrightarrow}{2}\end{array}$ & $\begin{array}{l}8 \\
8 \\
\ddot{g} \\
\dot{I}\end{array}$ & ลิ & $\underset{\substack{\infty \\
\infty}}{\stackrel{\infty}{\infty}}$ & 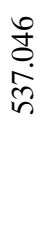 \\
\hline & 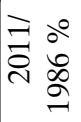 & 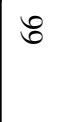 & in & $\hat{\sigma}$ & ๙ & $尺$ & న & $尺$ & $\approx$ \\
\hline & 을 & $\underset{\overbrace{}}{\widetilde{\sigma}}$ & 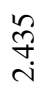 & $\begin{array}{l}\vec{\triangleright} \\
\stackrel{+}{+}\end{array}$ & $\begin{array}{l}\underset{J}{J} \\
\text { i }\end{array}$ & $\begin{array}{l}\infty \\
\stackrel{0}{0} \\
\stackrel{6}{0}\end{array}$ & ఫ̊ & $\underset{\infty}{\stackrel{d}{0}}$ & $\begin{array}{l}\frac{m}{\sigma} \\
\stackrel{0}{0}\end{array}$ \\
\hline 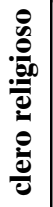 & $\frac{2}{2}$ & 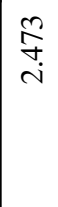 & $\underset{\stackrel{f}{\infty}}{\stackrel{m}{+}}$ & $\stackrel{\vec{N}}{r}$ & 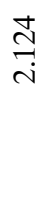 & $\begin{array}{l}\text { ふे } \\
\text { ¿े }\end{array}$ & $\stackrel{g}{\stackrel{g}{0}}$ & 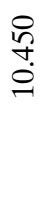 & $\begin{array}{l}\overrightarrow{8} \\
8 \\
8\end{array}$ \\
\hline \multirow{4}{*}{ 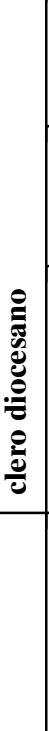 } & 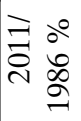 & $\nabla$ & 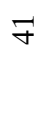 & $\mathscr{F}$ & $\stackrel{\circ}{\circ}$ & $\triangleright$ & gి & 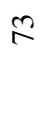 & $\stackrel{\infty}{\sim}$ \\
\hline & 을 & 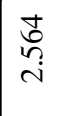 & 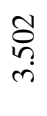 & $\begin{array}{l}\stackrel{\infty}{J} \\
\stackrel{+}{ \pm}\end{array}$ & $\begin{array}{l}\hat{\infty} \\
\stackrel{i}{ }\end{array}$ & 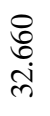 & $\begin{array}{l}\stackrel{\infty}{\mathscr{0}} \\
\stackrel{2}{i}\end{array}$ & $\begin{array}{l}\stackrel{\theta}{0} \\
\stackrel{0}{0} \\
\stackrel{0}{0}\end{array}$ & $\begin{array}{l}\hat{n} \\
\tilde{n} \\
m\end{array}$ \\
\hline & $\frac{2}{2}$ & $\begin{array}{l}0 \\
\text { ర్ } \\
\dot{r}\end{array}$ & $\begin{array}{l}\infty \\
\infty \\
\infty \\
\infty\end{array}$ & $\begin{array}{l}\text { ळે } \\
\infty \\
\text { ले }\end{array}$ & $\begin{array}{l}\stackrel{\infty}{\infty} \\
\dot{\infty}\end{array}$ & $\begin{array}{l}\stackrel{8}{\circ} \\
\infty \\
\dot{q}\end{array}$ & 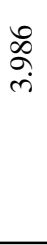 & $\begin{array}{l}\stackrel{n}{\sigma} \\
\stackrel{\Delta}{\Delta}\end{array}$ & $\stackrel{\infty}{\stackrel{\infty}{\Xi}}$ \\
\hline & & 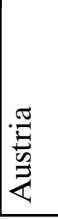 & $\begin{array}{l}\frac{0}{00} \\
\frac{00}{0} \\
\infty\end{array}$ & 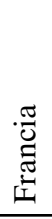 & 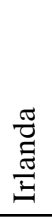 & $\stackrel{\overparen{\Xi}}{\stackrel{\Xi}{\Xi}}$ & 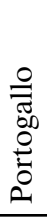 & $\begin{array}{l}\tilde{\Xi} \\
\tilde{\sigma} \\
\tilde{\sigma} \\
\text { की }\end{array}$ & 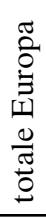 \\
\hline
\end{tabular}


Nel 2010 in Italia e in Portogallo queste sono, rispettivamente, il $63 \%$ ed il 67 \% di quante erano nel 1976, in Austria e Belgio il $35 \%$ e in Francia il $39 \%$. (iv) Nel caso del clero religioso il calo è generalizzato anche se con intensità non poco diverse. Si va dai pochi punti percentuali di Irlanda e Portogallo, al -25 \% di Italia e Spagna, ai casi più critici di Francia, Austria e Belgio dove - rispettivamente nel 2010 residua il $67 \%$, il $66 \%$ e appena il $50 \%$ del clero religioso presente nel 1976.

In sintesi estrema, e nell'ambito dei pochi casi considerati, si può dire che l'area francofona è quella cui si devono attribuire i risultati peggiori. D'altra parte, e in particolare per quello che riguarda la componente diocesana del personale ecclesiastico, il caso italiano è quello nel quale la crisi numerica, pure evidente, assume i toni meno aspri. Infine, qua e là è visibile qualche traccia di interventi sulle strutture della autorità religiosa: in Italia, ma anche in Francia.

Osservate a questo livello di generalità, è impossibile identificare le cause delle differenze internazionali appena segnalate. Non si sbaglia di certo, però, se si ipotizza che sia stata all'opera una serie molto nutrita di cause, tanto interne quanto esterne al sistema religioso locale. Entro i margini imposti dalla prudenza resa necessaria dalla povertà delle informazioni sugli altri casi nazionali, si può ragionevolmente avanzare una ipotesi. L'opera di centralizzazione e di verticalizzazione dell'autorità religiosa, di cui è stato protagonista il clero diocesano in Italia e particolarmente i vertici della Conferenza Episcopale Italiana, e il collegato effetto di bilanciamento che questa operazione ha costituito rispetto alle varie $d e$-formazioni della struttura della stessa autorità religiosa cattolica, potrebbe aver prodotto qualche effetto positivo in termini quantitativi. Se si sia trattato di mutazione genetica, di una supplenza che sta esaurendo la propria funzione, ${ }^{19} \mathrm{o}$ di qualcos'altro ancora, così come se si sia trattato di una scelta che aveva oppure che non aveva alternative realistiche, è problema che in questa sede non deve essere lasciato aperto.

\section{Conclusioni}

Per riassumere, si può ben dire che la struttura dell'autorità religiosa cattolica in Italia, rispetto agli inizi degli anni ' 70 del Novecento, presenta oggi alcune significative de-formazioni. (i) Ha subìto un processo di marcata centralizzazione e verticalizzazione. (ii) Ha perso non tanto in capillarità quanto in robustezza delle sue articolazioni territoriali soprattutto a causa del declino del personale, forse non solo di carattere quantitativo. (iii) La struttura dell'autorità ha poi anche

19 Si pensi ad esempio alle richieste che il nuovo Pontefice, sin dai suoi primi contatti con l'episcopato italiano, ha formulato di alcune profonde revisioni della struttura ecclesiastica in Italia: dalle regole per la selezione dei vertici, al numero delle diocesi, al ruolo delle conferenze episcopali regionali. 
subìto un processo di moltiplicazione e di diversificazione dei propri canali con effetti rilevanti, a partire da una crisi della tipica distinzione ecclesiale tra autorità e carisma e in particolare del ruolo del vescovo diocesano. (iv) Ha affievolito la propria funzione di promozione e di sostegno all'apostolato specifico dei laici e a quello dei religiosi, perseguendo più spesso una accentuata e ipertrofica egemonia della "pastorale", ovvero del ruolo e delle prospettive dei vescovi e clero diocesano.

Per valutare il grado di avanzamento e l'eventuale reversibilità o correggibilità di queste $d e$-formazioni sarà necessaria un'analisi approfondita che le metta in relazione con le profonde trasformazioni intervenute nello stesso periodo nell'ambiente in cui opera la struttura dell'autorità religiosa cattolica: tanto con riferimento all'ambiente sociale in generale quanto alla dimensione religiosa di questo stesso ambiente. Infatti, rispetto ai primi anni ' 70 del Novecento, globale e locale si intrecciano oggi in modi ormai profondamente diversi e il modello delle società imperniate sullo Stato (state societies) addirittura ha fatto in tempo ad entrare in profonda crisi e poi a tornare a suscitare qualche nostalgia. I processi di differenziazione sociale e di individuazione sono proceduti sino a soglie quaranta anni fa davvero poco immaginabili, e questo ha prodotto pressioni di secolarizzazione - quantitative e morfologiche - allora altrettanto raramente intuite. Nel frattempo il pluralismo religioso è divenuto una realtà anche nella società italiana (Pace ed. 2013).

Solo uno studio integrato (che, per quello che concerne la prospettiva disciplinare di questo saggio, significa di sociologia tout court e non solo di sociologia della religione) riuscirà a chiarire meglio in quale misura quelle de-formazioni dell'autorità religiosa cattolica in Italia sono state frutto di pressioni esterne e in quale misura di scelte interne. I risultati esposti consentono solo alcune provvisorie conclusioni. (i) Il vero e proprio progetto che ha guidato le politiche ecclesiastiche che hanno contribuito a quelle $d e$-formazioni (e che va inquadrato tra le strategie ecclesiastiche che si sono confrontate nel secondo post-Concilio) non è stato il frutto di una scelta obbligata. In parte non trascurabile si è affermato in alternativa a opzioni altrettanto realistiche. (ii) La grande varietà di situazioni regionali ${ }^{20}$ è anche riflesso di un margine di manovra che è costantemente rimasto a disposizione (ma solo di rado compreso ed ancor più raramente sfruttato) tanto dei livelli diocesani quanto dei livelli regionali della struttura dell'autorità religiosa cattolica in Italia, oltre che di quelli nazionali. Tra l'altro lo testimonia il grado diverso con cui - anche situazioni regionali simili - si è fatto ricorso a politiche non convenzionali di reclutamento del clero, alla parziale sostituzione della parrocchia con unità pastorali, a una compressione dello specifico ruolo del laicato da parte della pastorale e delle sue organizzazioni. (iii) Nel periodo considerato si è prodotta una sorta di parziale "meridionalizzazione" del cattolicesimo religioso italiano, dopo quasi un secolo in cui il ruolo guida era stato svolto

20 Almeno in parte analizzata in Diotallevi 2014c. 
dai cattolicesimi dell'Italia settentrionale. Lo testimonia la crescita del controllo clericale o "para-clericale" (spesso "operatori pastorali" in regime di lavoro dipendente) sulla vita ecclesiale che si manifesta tra l'altro nell'ipertrofia delle "pastorali". Lo rivela la perdita di influenza della forma-chiesa e una riduzione delle influenze extra-religiose del cattolicesimo italiano. Lo rivela infine una maggiore dipendenza delle forme dell'offerta religiosa "cattolica" dalle istanze della domanda, con la conseguenza di una maggiore diversificazione e di una maggiore competizione tra attori diversi dell'offerta religiosa "cattolica" e gli ancora maggiori rischi connessi di de-ecclesializzazione (Marzano Pace eds. 2013). In tutto questo credo possano facilmente essere letti i segni del rischio di cedimento al modello di una religione a bassa intensità ("low intensity religion", Bryan S. Turner). (iv) Il periodo analizzato appare come quello della elaborazione e del perseguimento di un progetto "neoclericale" (a conti fatti diverso da quelli del passato perché più debole, tanto sul fronte interno quanto su quello esterno). Questo "neoclericalismo debole" può vantare non trascurabili successi se lo si considera in uno scenario di comparazione internazionale, ma anche insuccessi, se si considerano i problemi cui intendeva dare quella risposta che riteneva - non senza ragioni - fosse mancata nel primo post-Concilio. Oggi questo "neoclericalismo debole" appare una sorta di supplenza, che si sta protraendo da alcuni decenni. La sua realizzazione ha richiesto un massiccio investimento di risorse (di ogni tipo) ed il suo principale risultato, probabilmente, è stato quello di guadagnare tempo ed insieme esporsi il cattolicesimo italiano a rischi forse imprevisti. Così, mentre questa supplenza esaurisce la propria forza e tende a degenerare, si impone una domanda. Restano energie sufficienti per mettere a frutto positivamente il tempo guadagnato? $\mathrm{E}$ ancora: la risposta che verrà a questa domanda sarà simile nelle diverse aree della penisola?

\section{Fonti statistiche utilizzate}
[A] Annuarium Statisticum Ecclesiae.
[C] Azione Cattolica Italiana.
[E] GfK Eurisko, I valori del sovvenire, programma di ricerca con cadenza biennale
[G] Istituto Centrale per il Sostentamento del Clero
[I] Notiziari CEI

\section{Bibliografia}

Abbott, Andrew: The System of Professions. An Essay on the Division of Expert Labor. Chicago 1988.

Acerbi, Antonio: "La Chiesa italiana e Giovanni Paolo II", in: Acerbi A. / Frosini G.: Cinquant'anni di Chiesa in Italia. Bologna 2006, pp. 67-114.

Acquaviva, Sabino S.: L'eclissi del sacro nella società industriale. Milano 1961. 
Alberigo, Giuseppe: Il cristianesimo in Italia. Bologna 1992.

Ammerman, Nancy T.: Sacred Stories, Spiritual Tribes. Finding Religion in Everyday Life. Oxford 2014.

Beckford, James A.: Social theory and religion. Cambridge 2003.

Beyer, Peter: Religion in the Context of Globalization. London 2013.

Borghesi, Claudio / Castegnaro, Alessandro / Dal Piaz, Giovanni / De Sandre, Italo: Giovani e vita consacrata. Padova 2007.

Bressan, Luca: "Preti nel nuovo millennio. Alcuni dati di una recente inchiesta sui preti e sui seminaristi", in: La Scuola Cattolica (134) 2006, pp. 393-436.

Brunetta Giuseppe: "Il clero in Italia dal 1888 al 1989", in: Polis (3) 1991, pp. 423-450.

Burgalassi, Silvano: Le cristianità nascoste. Bologna 1970.

Cesareo, Vincenzo (e al.): La religiosità in Italia. Milano 1995.

Chaves, Mark: "Denominations as Dual Structures: an Organizational Analysis", in: Sociology of religion (54) 1993, pp. 147-169.

Ders.: "Secularization as Declining Religious Authority", in: Social forces 1994, pp. 749_ 774.

Ders.: Congregations in America. Cambridge 2004.

Ders.: "All Creatures Great and Small: Megachurches in Context", in: Review of Religious Research (47/4) 2006, pp. 329-346.

Ders.: American Religion. Princeton 2011.

Coleman, John A.: The Evolution of Dutch Catholicism, 1958-1974. Berkeley 1978.

Consorti, Pierluigi: Diritto e religione. Bari 2010.

Dalpiaz, Giovanni: "La vie religieuse en Italie", in: Social compass (60) 2013, pp. 332347.

Demerath, Nicolas J. III: "Secularization and Sacralization. Deconstructed and Reconstructed", in: Beckford J.A. / Demerath, Nicolas J. III (ed.): The Sage Handbook of the Sociology of Religion. London 2007, pp. 57-80.

De Rosa, Gabriele / Gregory, Tullio: La storia dell'Italia religiosa, vol. III. Roma - Bari 1993.

DiMaggio, Paul J.: "La gabbia di ferro rivisitata. Isomorfismo istituzionale e razionalità collettiva nei campi organizzativi", in: Powell, Walter W. / DiMaggio, Paul J.: Il neoistituzionalismo nell'analisi organizzativa. Torino 2000, pp. 88-115.

Diotallevi, Luca: Religione, Chiesa e modernizzazione: il caso italiano. Roma 1999.

Ders.: Il rompicapo della secolarizzazione italiana. Soveria Mannelli 2001.

Ders.: "Internal Competition in a National Religious Monopoly: the Catholic Effect and the Italian case", in: Sociology of Religion (63/2) 2002, pp. 345-364.

Ders.: "Meno siamo, meglio stiamo? Il referendum sulla fecondazione assistita e il peso del fattore religioso", in: Polis (3) 2007, pp. 489-515.

Ders.: "Chiesa e cattolici nell'Umbria di oggi: alcuni adattamenti inutili", in: Angeletti, Silvia / Armillei, Giorgio (a cura di): Poliarchia e bene comune. Chiesa, economia e politica per la crescita dell'Umbria. Bologna 2010a, pp. 179-205.

Ders.: "Diözesen und Säkularisierung in Italien. Eine Hypothese zum Verständnis des Strukturwandels in der Katholischen Kirchen nach dem Zweiten Vatikanischen Konzil", in: Damberg, Wilhelm / Hellemans, Staf (Hrsg.): Die neue Mitte der Kirche. Der Aufstieg der intermediären Instanzen in den europäischen Großkirchen seit 1945. Stuttgart 2010b, S. 179-214. 
Ders.: "Clero in trasformazione. Il caso italiano", in: Rivista del clero italiano vol. XCV 2014a, pp. 754-773.

Ders.: "Il cattolicesimo italiano agli inizi del XXI secolo come 'religione a bassa intensità'. Una trasformazione ancora in corso e non ancora irreversibile", in: Angelini, Giuseppe / Bressan, Luca / Diotallevi, Luca / Givone, Sergio / Guanzini, Isabella / Rizzi, Marco / Sequeri, Pierangelo / Ubbiali, Sergio: Una fede per tutti? Forma cristiana e forma secolare. Milano 2014b, pp. 97-156.

Ders.: "Trasformazioni della struttura della autorità religiosa cattolica in Italia", in: Istituto della Enciclopedia Treccani. L'Italia e le sue regioni. Istituzioni, Territori, Culture, Società, vol. III. Roma 2014c.

Ders. (a cura di): La parabola del clero. Uno sguardo socio-demografico sui sacerdoti diocesani in Italia. Torino 2005.

Favale, Agostino (et al.): Movimenti ecclesiali contemporanei. Roma 1991.

Finke, Roger: "Innovative Returns to Tradition: Using Core Teachings as the Foundation for Innovative Accommodation", in: Journal for the Scientific Study of Religion (43/1) 2004, pp. 19-35.

Finke, Roger / Wittberg Patricia: "Organizational Revival from Within: Explaining Revivalism and Reform in the Roman Catholic Church", in: Journal for the Scientific Study of Religion (39/2) 2000, pp. 154-170.

Garelli, Franco: Religione e Chiesa in Italia. Bologna 1991.

Garelli, Franco / Guizzardi, Gustavo: Un singolare pluralismo. Bologna 2003.

Gorski, Philip S. / Altinordu, Ateş: “After Secularization?”, in: Annual Review of Sociology (34) 2008, pp. 55-85.

Luhmann, Niklas: Organizzazione e decisione. Milano 2005.

Marzano, Marco / Pace, Enzo (eds.):"The many faces of Italian Catholicism in the 21st century", in: Social compass, (60/3) 2013, pp. 299-361.

McLeod, Hugh: Religion and the People of Western Europe 1789-1989. Oxford 1997.

Ders.: Secularisation in Western Europe, 1848-1914. New York 2000.

Ders.: The religious crisis of the 1960s. Oxford 2007.

Melloni, Alberto: Tutto e niente. I cristiani d'Italia alla prova della storia. Roma - Bari 2013.

Moro, Renato: "Il 'modernismo buono", in: Storia contemporanea (4) 1988, pp. 625-716.

Molina, Stefano: "Il clero diocesano in Italia: uno sguardo al presente e al recente passato", in: Diotallevi, Luca (Hg.): La parabola del clero. Uno sguardo socio-demografico sui sacerdoti diocesani in Italia. Torino 2005, pp. 25-14.

Nesbitt, Paula: "Keepers of the Tradition: Religious Professionals and their Careers", in: Beckford J.A. / Demerath N.J. III (ed.): The Sage Handbook of the Sociology of Religion. London 2007, pp. 295-322.

Pace, Enzo (ed.): Le religioni nell'Italia che cambia. Mappe e bussole. Roma 2013.

Prodi, Paolo: Il paradigma tridentino. Un'epoca di storia della Chiesa. Brescia 2010. Putnam, Robert D. / Campbell, David E.: American Grace. How Religion Divides and Unites us. New York 2010.

Rinallo, Diego / Borghini, Stefania / Bamossy, Gary: "When Sacred Objects Go B ${ }^{\circledR} a(n) d$ : Fashion Rosaries and the Contemporary Linkage of Religion and Commerciality", in: Rinallo, Diego / Scott, Linda / Maclaran, Pauline (ed.): Consumption and Spirituality. London 2013, pp. 29-40. 
Simmel, Georg: La differenziazione sociale. Roma - Bari 1982.

Stark, Rodney / Iannaccone, Laurence R.: "A Supply-Side Reinterpretation of the 'Secularization' of Europe", in: Journal for the scientific study of religion (33/3) 1994, pp. 230-252.

Turner, Bryan S. (ed.): Secularization, voll. I-IV. Los Angeles 2010.

Ders.: Religion and Modern society. Cambridge 2011.

Ders.: "Post-Secular Society: Consumerism and the Democratization of Religion", in: Gorski, Philip S. / Kim, David K. / Torpey, John / Van Antwerpen, Jonathan (ed.): The Post Secular in Question. Religion in contemporary society. New York 2012, pp. 135185.

Weber, Max: Economia e società, vol. I. Torino 1995.

Wittberg, Patricia: The Rise and Fall of Catholic Religious Orders. New York 1994.

Wuthnow, Robert: Restructuring of the American religion: Society and Faith since World War II. Princeton 1988. 